\title{
Transatlantica
}

Revue d'études américaines. American Studies Journal

\section{Alina Szapocznikow : La mémoire à l'œuvre}

\section{Sophie Mayer}

\section{(2) OpenEdition}

Journals

Édition électronique

URL : https://journals.openedition.org/transatlantica/6670

DOI : $10.4000 /$ transatlantica.6670

ISSN : 1765-2766

Éditeur

Association française d'Etudes Américaines (AFEA)

Édition imprimée

Date de publication : 31 décembre 2013

\section{Référence électronique}

Sophie Mayer, «Alina Szapocznikow : La mémoire à l'œuvre », Transatlantica [En ligne], 2 | 2013, mis en ligne le 04 avril 2014, consulté le 31 janvier 2023. URL : http://journals.openedition.org/ transatlantica/6670 ; DOI : https://doi.org/10.4000/transatlantica.6670

Ce document a été généré automatiquement le 31 janvier 2023.

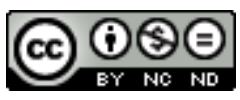

Creative Commons - Attribution - Pas d'Utilisation Commerciale - Pas de Modification 4.0 International - CC BY-NC-ND 4.0

https://creativecommons.org/licenses/by-nc-nd/4.0/ 


\title{
Alina Szapocznikow : La mémoire à l'œuvre
}

\author{
Sophie Mayer
}

1 Alina Szapocznikow (1926-1973), sculptrice, dessinatrice et plasticienne polonaise, née à Kalisz dans une famille bourgeoise juive non pratiquante, a joui de son vivant d'une notoriété considérable en Pologne, mais aussi en France, après l'immédiate rétrospective que lui consacra le Musée d'Art Moderne de la ville de Paris en 1973, quelques jours après sa mort, à l'initiative du critique d'art Pierre Restany.

2 Ce n'est toutefois qu'en 1998, à l'occasion du $25^{\text {5ème }}$ anniversaire de sa mort et grâce à la persévérance de son fils Piotr Stanislawski, d'Anda Rottenberg (historienne et critique d'art polonaise influente qui dirige la Galerie Zacheta à Varsovie) qu'une exposition monographique et une tournée furent organisées, qui permirent de redécouvrir le travail de l'artiste. De nombreux musées polonais acquirent alors ses œuvres.

3 En 2012 et en 2013, l'œuvre de Szapocznikow était sur tous les fronts : une rétrospective intitulée "Alina Szapocznikow: Sculpture Undone» fut organisée au MoMa de New York, en partenariat avec le centre d'art bruxellois Wiels et le Hammer Museum de Los Angeles; le centre Georges Pompidou lui consacra une importante rétrospective intitulée "Alina Szapocznikow : du dessin à la sculpture »; enfin, ses œuvres créèrent l'événement à la FIAC, où l'artiste était représentée par la galerie Loevenbruck, qui a largement contribué à sa (re-) mise en lumière. Ce n'est d'ailleurs peut-être pas un hasard si l'exposition que la galerie présenta à la fin de l'année 2013 s'intitulait « EEuvres lumineuses».

4 Alina Szapocznikow est aujourd'hui considérée comme une artiste féminine majeure du XXe siècle. Hervé Loevenbruck n'hésite pas à la placer aux côtés de la française Louise Bourgeois et de l'américaine d'origine allemande Eva Hesse. A l'occasion de sa présentation de l'artiste, le MoMa soulignait la façon dont elle réinventa et reconceptualisa la sculpture, en travaillant notamment à partir de matériaux non traditionnels, très peu chers et très peu utilisés (des matériaux utilisés dans les BTP), tels que la laine de verre, la résine de polyester et la mousse de polyuréthane. Ces matériaux, Szapocznikow les utilisa pour réaliser des expérimentations qui relevaient 
aussi bien du Nouveau Réalisme, du Pop Art et du Surréalisme que d'une volonté d'inventer un nouveau rapport au corps et à la temporalité. Elle entreprit ainsi de détourner, en se les réappropriant, des objets du quotidien et de la grande consommation: elle créa des bouches-lampes fabuleuses et des têtes-cendriers inquiétants (voir Illustrations $\mathbf{n}^{\circ} \mathbf{1}$ et $\mathbf{n}^{\circ} 2$ ), photographia le devenir informe d'un chewing-gum après l'avoir mâché et sculpté dans sa bouche (inaugurant une sorte de Land Art miniature et organique, voir Illustration $\mathbf{n}^{\circ} 3$ ), projeta de réaliser une série de sculptures immenses de Rolls Royce (échelle 2) pour la documenta de Harald Szeeman ${ }^{1}$, ou encore nourrit le projet (jamais abouti) de bâtir une patinoire artificielle dans le cratère du Vésuve. Elle conçut par ailleurs des installations plastiques pour le moins singulières puisque celles-ci mettaient en scène des parties de son propre corps, moulées puis retravaillées à partir de ses matériaux de prédilection. C'est là ce qui retient particulièrement l'attention.

En effet, si les expérimentations esthétiques de Szapocznikow s'inscrivirent dans l'intense période de créativité artistique de l'après-seconde guerre mondiale et étaient en avance sur leur époque - préfigurant à maints égards les innovations de futurs artistes (les artistes du Land Art mais également des artistes comme Peter Buggenhout ou Magdalena Abakanowicz) - elles engagèrent simultanément un travail sur la mémoire, un travail de mémoire, qui la distinguaient de ses contemporains. C'est ce qu'atteste la façon dont le corps et la matière sont travaillés et représentés dans les œuvres: qu'ils aient été moulés à partir de modèles vivants, du propre corps de l'artiste, ou bien inspirés de représentations antiques et classiques, les corps sculptés sont presque toujours morcelés, fragmentés, amputés, défigurés et atteints dans leur intégrité. Ce dé-membrement, cette dispersion 'métonymique', ont pour effet de créer chez le spectateur un sentiment ambivalent, chargé de malaise. En effet, tout en célébrant la puissance de la vie et de la création, les œuvres introduisent des images associées à la violence, à la destruction et à la mort. Si les parties du corps privilégiées par l'artiste sont empreintes de sensualité et de féminité, évoquant la fertilité et la création - comme par exemple ses bouches-lampes (voir Illustration $\mathbf{n}^{\circ} \mathbf{1}$ ), ses seinsdesserts et ses ventres-coussins - il émane des sculptures quelque chose de l'ordre du 'monstrueux'. Et ce 'monstrueux' ne relève pas seulement d'une esthétique que l'on pourrait qualifier de baroque, de burlesque ou de surréaliste, et qui s'inspire du fabuleux et du mythologique : il relève également de l' 'Unheimliche', de l'Uncanny' un sentiment difficile à cerner de prime abord mais que plusieurs œuvres assez 'explicites' viennent renforcer, qui font basculer du côté de la hantise et de la mort. Dans l'univers de Szapocznikow, la beauté côtoie l'horreur, l'horreur vient inquiéter la beauté, la puissance de vie et de création, de composition, est menacée par la destruction, par une dé-composition sourde et menaçante. C'est le cas de ses « Herbiers » (voir Illustration $\mathbf{n}^{\circ} 4$ ) et de ses installations mortuaires ( «' L'enterrement d'Alina " par exemple), dans lesquels l'artiste met en scène sa propre sépulture fantasmée, utilisant des vêtements et des photos intimes qu'elle mélangeait et retravaillait dans la résine de polyester ou la mousse de polyuréthane, et qui donnent l'impression d'une vraie sépulture exhumée. Cette démarche - que l'on pourrait rapprocher des masques mortuaires de Marcel Duchamp ou des projections posthumes d'Emily Dickinson - soulèvent des questions d'ordre philosophique et eschatologique essentielles : celle de la survivance de et par la création, ainsi que la part de la mémoire dans le processus créatif. 
6 Un retour sur l'histoire et la biographie de l'artiste s'impose si l'on veut saisir pleinement les implications d'un travail dont les expérimentations formelles et esthétiques semblent indissolublement liées à une réflexion non seulement sur les possibilités qu'offrent l'art 'plastique' et la sculpture mais également sur le rôle de la mémoire dans la démarche de création - la mémoire enfouie du corps et sa possible résurgence dans le 'corps' de l'œuvre. Szapocznikow a en effet côtoyé la mort dès son plus jeune âge : sa mère, son frère et elle furent internés en 1940 dans le ghetto de Pabianice puis transférés en 1942 dans le ghetto de Lodz, pour être ensuite déportés à Bergen-Belsen via Auschwitz, où Szapocznikow assista sa mère en tant qu'aideinfirmière dans les hôpitaux du camp. C'est pendant ces années qu'elle contracta une tuberculose péritonéale qui la rendit stérile à l'âge de 23 ans. Plus tard, à l'âge de 43 ans, elle se vit diagnostiquer un cancer du sein. L'objectif n'est pas ici de réduire l'œuvre à une lecture traumatique, mais d'interroger la possible résonance de celle-ci avec une expérience vécue dont elle semble porter la trace, les 'stigmates'. Comment ne pas voir, en effet, dans l'obsession des détails anatomiques et organiques, dans l'intégration de photos de camps de concentration et de charniers (agrandies et placées dans de grands blocs de résine, où les visages et les corps donnent l'impression d'avoir été figés dans la glace et dans le temps), dans les représentations posthumes (Szapocznikow 'survécut' à l'horreur des camps quand d'autres, comme son frère, $\mathrm{y}$ laissèrent leur vie), ou encore dans les séries de 'tumeurs' (sortes de perles ou de larmes de seins et de visages de madones qui prolifèrent et se réverbèrent à l'infini), le travail de la mémoire à l'œuvre? La maladie et la mort hantent jusqu'au matériau des œuvres, avec leurs effets de charpie, de peaux et d'os, de chaux et de cire funéraire.

7 Les expérimentations plastiques de Szapocznikow semblent avoir été nourries et travaillées de l'intérieur par une mémoire dont la temporalité est 'double' - aussi bien 'rétrospective' (de l'ordre de la résurgence et du souvenir) que 'prospective' (de l'ordre de l'anticipation, de la prémonition) : ses multiples représentations de la maladie et de la mort apparaissent comme les incarnations allégoriques d'un mal inextirpable, d'une mémoire tumorale gangrénée aussi bien par la re-convocation d'expériences passées que par la prescience d'expériences à venir. En ce sens, Szapocznikow était à la fois en retard et en avance sur sa mort, prise dans l'étau d'un présent urgent, qui célébrait frénétiquement la vie pour tenter de conjurer la disparition. Se pose alors une question essentielle: celle du potentiel cathartique de l'œuvre d'art, sa puissance de 'conjuration' - de cicatrisation, peut-être. On peut en effet s'interroger sur la signification du démembrement et de la dé-composition à l'œuvre dans les sculptures et les installations de l'artiste : ils pourraient souligner l'impossible travail de mémoire, de re-composition et de re-membrement, ou bien signaler tout au contraire la possible re-composition, le possible re-membrement - de son corps, sa mémoire, son identité - à travers un travail de dé-composition volontaire et maîtrisé.

8 Alina Szapocznikow nous invite ainsi à réfléchir à la relation entre l'œuvre d'un artiste et son environnement de production, son histoire, et soulève des questions fondamentales quant au travail de la mémoire - et sur la mémoire - qu'engage tout processus de création. La question de l'art en tant qu'empreinte et que 'séquelle' (aussi bien esthétique, physique et psychologique), ainsi que le potentiel cathartique de l'œuvre, sont au cœur de ses expérimentations plastiques. Le travail sur le corps devient ainsi le théâtre d'une confrontation violente entre soi et soi mais aussi, plus largement, l'arène où s'affrontent la grande histoire et la petite histoire. Et ce n'est pas 
limiter la portée de l'œuvre de Szapocznikow que d'apporter des éléments contextuels et biographiques pour l'éclairer: au contraire, ceux-ci rendent compte de façon d'autant plus saisissante de la manière dont l'artiste puisa - consciemment ou non dans son expérience vécue, pour sublimer (conjurer, peut-être) une souffrance aussi bien personnelle que collective.

\section{NOTES}

1. La documenta est une manifestation d'art contemporain qui a lieu tous les cinq ans à Cassel, en Allemagne, depuis sa création en 1955 par Arnold Bode. Harald Szeeman fut le commissaire d'exposition de la documenta en 1972. 